\title{
Uric acid as a marker of clinical severity and comorbidities in plaque psoriasis
}

\author{
Adameck Abraham Hernandez-Collazo, ${ }^{1}$ Guadalupe Villanueva-Quintero, ${ }^{1}$ \\ Marco Antonio Rodríguez-Castellanos, ${ }^{1}$ Eduardo David Poletti-Vázquez ${ }^{2}$ and Anabell Alvarado-Navarro ${ }^{3}$ \\ ${ }^{1}$ Instituto Dermatológico de Jalisco Dr. José Barba Rubio, Jalisco, México; ${ }^{2}$ Universidad Autónoma de Aguascalientes, Aguascalientes; ${ }^{3}$ Center of \\ Immunology and Dermatology Research, Jalisco, Mexico
}

\begin{abstract}
Introduction: Hyperuricemia is common in patients with psoriasis. Uric acid elevation in psoriasis has been suggested to be strongly associated with cardiovascular morbidity. Objective: To determine the relationship between uric acid levels and clinical severity as measured by the Psoriasis Area Severity Index (PASI), body surface area (BSA) and static Physician's Global Assessment (SPGA) in patients with plaque psoriasis and nail comorbidities and psoriatic arthritis. Method: Determination of serum uric acid in 45 patients with plaque psoriasis and 45 controls matched by gender, age and body mass index; measurement of patient clinical severity indices and presence of nail and joint manifestations. Results: Patients with psoriasis had higher levels of uric acid (7.03 \pm 1.47 versus $5.32 \pm 1.17, p<0.01)$, and higher prevalence of asymptomatic hyperuricemia than controls (68\% versus $17.8 \%, p<0.01)$. There was significant proportional correlation between PASI-determined severity and uric acid $\left(r^{2}=0.70\right)$, and between joint manifestations and hyperuricemia in patients with plaque psoriasis ( $p<0.01$; $O R=2.85,95 \% \mathrm{Cl}=1.52-5.33)$. Conclusions: Serum uric acid levels had a proportional correlation with PASI and were associated with joint manifestations in patients with plaque psoriasis.
\end{abstract}

KEY WORDS: Hyperuricemia. Psoriasis. Psoriatic arthritis. Uric acid.

\section{Introduction}

Psoriasis is a chronic autoinflammatory disease that affects $2 \%$ of the population in Western countries. ${ }^{1,2}$ Its pathogenesis includes an immune cascade of pro-inflammatory cytokines that results in a state of persistent inflammation and increased epidermal cell turnover. ${ }^{3}$ Its spectrum includes different cutaneous manifestations that can coexist in a single patient, although they all share essential characteristics: erythema, thickening and scales. The persistent inflammatory state has been associated with extracutaneous manifestations: increased cardiovascular risk, psoriatic arthritis secondary to oxidative stress increase and antioxidant capacity decrease. Epidermal turnover is accentuated, with the clinical and diagnostic histopathological features of the disease ${ }^{4,5}$ The increase in the keratinocyte cell cycle induces an increase in purine metabolism, which elevates serum uric acid levels. ${ }^{6}$

Since 1948, Lobitz and Brunsting described for the first time the relationship between hyperuricemia and psoriasis; ${ }^{7}$ recently, Gisondi et al. ${ }^{8}$ confirmed this relationship, identifying psoriasis as the most important risk factor in a multivariate analysis when compared with other known risk factors for hyperuricemia, such as obesity or metabolic syndrome. In 2016, Li iet al., in a meta-analysis that assessed data from 14 clinical trials and included 29416 patients with psoriasis, concluded that the correlation between psoriasis and hyperuricemia shows an ethnic pattern associated with genetic components and different lifestyles. ${ }^{9}$

The increase in serum uric acid has been associated with an increase in the carotid intima thickness in patients with psoriatic arthritis, whereas in patients
Correspondence:

Adameck Abraham Hernández-Collazo

E-mail: adam_ck11@hotmail.com
Date of reception: 21-06-2017

Date of acceptance: 09-11-2017

DOI://dx.doi.org/10.24875/GMM.M18000168
Gac Med Mex. 2018;154:357-361

Contents available at PubMed www.gacetamedicademexico.com 
without psoriasis it has been used as a predictor of cardiovascular events and cardiovascular-origin mortality. ${ }^{10}$ However, to date, whether the relationship between uric acid and cardiovascular events in psoriasis is circumstantial or causal is not known.

The investigation of hyperuricemia as a parameter of prognosis and clinical assessment in psoriasis is promising. Isha et al. demonstrated that effective treatment of psoriasis for 12 weeks significantly reduces uric acid and C-reactive protein serum levels; ${ }^{11}$ however, thus far there are no studies evaluating the correlation between uric acid and clinical severity in patients with psoriasis.

The purpose of this study was to establish the association between uric acid levels with the severity scales that are most widely used in clinical practice, such as the Psoriasis Area and Severity Index (PASI), Body Surface Area (BSA) and static Physician Global Assessment (SPGA), in addition to their association with other clinical severity parameters such as dyslipidemia and joint and nail manifestations. In addition, uric acid levels and the prevalence of hyperuricemia were compared in patients with psoriasis and control subjects.

\section{Methods}

Forty-five patients that attended the psoriasis clinic of the Dr. José Barba Rubio Dermatological Institute of Jalisco for the first time were consecutively recruited between November 2014 and January 2015. All patients with clinical and histopathological diagnosis of psoriasis and who did not receive treatment for at least 3 months prior to the investigation were included. The control group was composed of 45 healthy people, selected to be paired according to age, gender and body mass index with the psoriasis patients. Both in the control group and in the patient group, individuals with consumption of $>2$ drinks per week, as assessed through a standardized questionnaire, high-protein or catabolic diets, advanced chronic kidney disease, medications that alter uric acid metabolism (allopurinol, diuretics, ethambutol, pyrazinamide, ketoconazole, salicylates, or theophylline) and a family or personal history of gout were not included.

Each patient was evaluated by a dermatologist to measure clinical severity using PASI, BSA and SPGA, and assessed for psoriatic arthritis diagnosis using the Classification Criteria for Psoriatic Arthritis (CASPAR). $B S A$ is based on the measurement of the surface affected by psoriatic lesions using the palm of the patient's hand (fingers included), which is equivalent to $1 \%$ of total body surface. ${ }^{12}$
sPGA determination employs a subjective scale that scores lesion severity without evaluating the extent, with a 0-to- 6 scale; the lowest value is applied to the absence of lesions or "clear" and the score increases with infiltration, erythema and scale, which is identified with the highest value. ${ }^{13}$

The PASI index is established with the assessment of each psoriatic lesion, by assigning a value of 0 to 4 to each of the 3 characteristics of the elemental lesion according to its severity: erythema, infiltration and scale. This result is combined with a weighted evaluation of the affected area of each segment of the body: head and neck, trunk, upper and lower extremities.12,13

Regarding the CASPAR psoriatic arthritis classification criteria, the presence of peripheral or axial arthropathy or enthesitis and three points of any of the clinical, serological or radiological manifestations of the disease must be confirmed in the patient. ${ }^{14}$

The presence of nail manifestations was determined in patients with psoriasis: onycholysis, pitting, leukonychia, oil drop and salmon patch. A $3.5 \mathrm{~mL}$ peripheral blood sample after a 12-hour fasting was obtained both from patients and controls for serum uric acid determination by means of SpectrAA-200 Varian spectrophotometer. Hyperuricemia was defined in individuals with uric acid serum values higher than $6.5 \mathrm{mg} / \mathrm{dL}$ for the male, and $6.0 \mathrm{mg} / \mathrm{dL}$ for female gender.

Measures of central tendency, descriptive statistics, Student's t-test and Pearson's correlation were used by means of the statistical program OpenEpi, version 3.01.

\section{Results}

A sample of 45 patients and 45 healthy controls matched by age, gender and body mass index was obtained. In both groups there were 29 men and 16 women; mean age was $49.38 \pm 17.36$ years in the patient group and $50.98 \pm 17.3$ years in the controlgroup; body mass index in patients had a mean of $31.18 \pm$ $6.66 \mathrm{~kg} / \mathrm{m}^{2}$ and in the control group $30.15 \pm 6.08 \mathrm{~kg} / \mathrm{m}^{2}$. Epidemiological characteristics are described in table 1. In the patients with psoriasis, the statistical mean for PASI was $10.90 \pm 5.82$, for BSA $28.26 \pm$ $15.26 \%$ and for SPGA $2.96 \pm 2.38$. Of the 45 patients, 6 did not attend the outpatient clinic for psoriatic arthritis diagnosis or attended without hands or feet X-rays or serum rheumatoid factor; 39 were assessed for classification, out of which $53 \%$ (21/39) had joint manifestations that met the CASPAR criteria.

Significantly, patients with psoriasis had higher levels of uric acid than subjects in the control group 
Table 1. Demographic, somatometric and biochemical characteristics in individuals with and without psoriasis

\begin{tabular}{|c|c|c|}
\hline Group & $\begin{array}{l}\text { Psoriasis } \\
(n=45)\end{array}$ & $\begin{array}{l}\text { Control } \\
(n=45)\end{array}$ \\
\hline Men/women ratio (n) & $29 / 16$ & 29/16 \\
\hline \multirow{2}{*}{$\begin{array}{l}\text { Hyperuricemia } \\
\text { frequency (n) }\end{array}$} & $31(68.88 \%)$ & $8(17.88 \%)$ \\
\hline & Media \pm SD & Media \pm SD \\
\hline Age (years) & $49.38 \pm 17.36$ & $50.98 \pm 17.3$ \\
\hline Body mass index $\left(\mathrm{kg} / \mathrm{m}^{2}\right)$ & $31.18 \pm 6.66$ & $30.15 \pm 6.08$ \\
\hline Serum uric acid (mg/dL) & $7.03 \pm 1.47$ & $5.32 \pm 1.17$ \\
\hline
\end{tabular}

$(7.03 \pm 1.47 \mathrm{mg} / \mathrm{dL}$ versus $5.32 \pm 1.17 \mathrm{mg} / \mathrm{dL}, \mathrm{p}<0.01)$. Hyperuricemia frequency was higher in the psoriasis than in the control group (68.88 versus $17.8 \%, p<$ 0.01 ), with a significant difference. These results were maintained when patients were stratified by gender; in this case, both men and women with psoriasis had higher uric acid than their control counterparts. Similarly, in the presence of metabolic syndrome, patients with psoriasis had higher uric acid levels than the control group.

Figure 1 shows that, with Pearson's correlation coefficient, a strong positive correlation was found between PASI measurement and uric acid serum levels $\left(r^{2}=70\right.$, $p<0.01$ ). Conversely, severity as measured by BSA $\left(r^{2}=0.32\right)$ and SPGA $\left(r^{2}=0.29\right)$ had a weakly positive correlation (Figures 2 and 3 ). As for other severity indices, $57.77 \%$ (26/45) of patients had nail manifestations, mainly onycholysis; out of them, $69 \%$ (18/26) had hyperuricemia. No significant association was identified with the contingency tables or the chi-square test $(p=0.43)$.

Table 2 shows in detail the variables studied in the group of patients with psoriasis who had hyperuricemia (31/45) versus those with uric acid normal levels with regard to gender and psoriasis (14/45). As for age, gender, body mass index, as well as total cholesterol or triglyceride levels, no statistical differences were found between the group with psoriasis and hyperuricemia versus patients with psoriasis and normal uric acid. Severity evaluated by PASI was higher in patients with hyperuricemia as compared to that of those with psoriasis without hyperuricemia (10.90 \pm 5.81 versus $5.41 \pm 2.76$ ), with the difference being significant $(p=0.007)$. Severity measured by BSA was higher in patients with hyperuricemia compared to that of those with psoriasis without hyperuricemia (28.26 \pm 19.05 versus $14.18 \pm 12.49$ ); however, this difference was not statistically significant between both groups

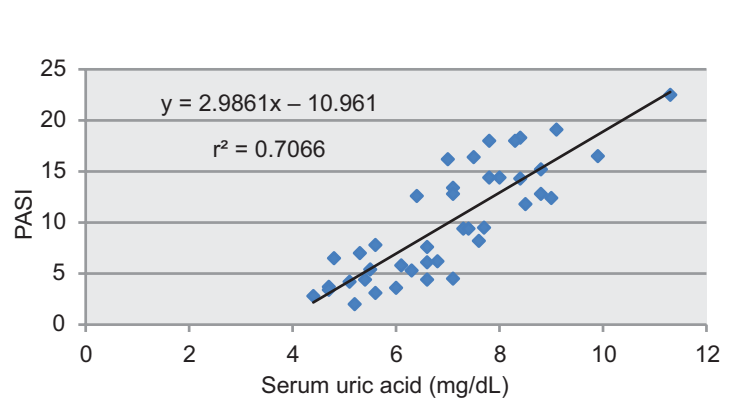

Figure 1. Pearson's correlation. PASI and serum uric acid $\left(r^{2}=0.70\right.$, $p<0.01) . P A S I=$ Psoriasis Area and Severity Index.

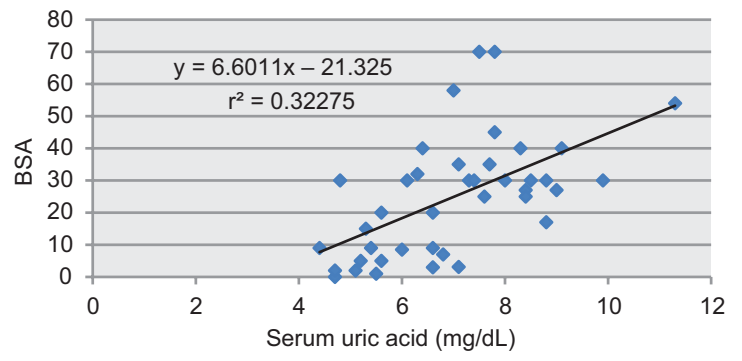

Figure 2. Pearson's correlation. $B S A$ and serum uric acid $\left(r^{2}=0.32\right.$, $p<0.01)$. BSA = Body Surface Area.

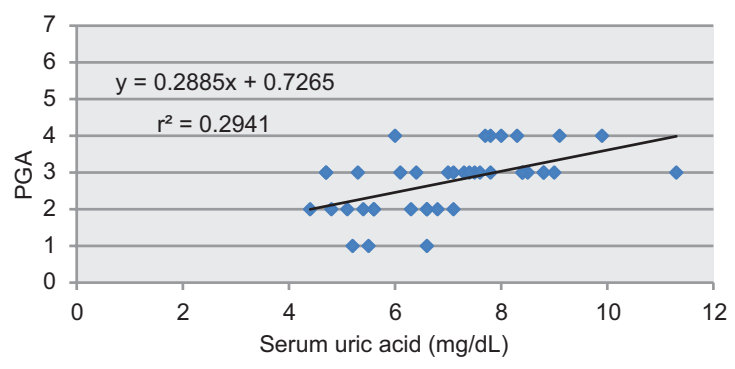

Figure 3. Pearson's correlation. $s P G A$ and serum uric acid $\left(r^{2}=0.29\right.$, $p<0.01) . s P G A=$ Static Physician's Global Assessment.

$(p=0.16)$. In addition, severity measured by SPGA did not show any significant difference between both groups $(2.97 \pm 0.76$ versus $2.21 \pm 0.80, p=0.95)$.

Finally, $81 \%$ of patients with joint manifestations that met the CASPAR criteria exhibited hyperuricemia (17/21). Through the same statistical analysis, a statistically significant association was identified between the presence of psoriatic arthritis and hyperuricemia $(p<0.01, \mathrm{OR}=2.85,95 \% \mathrm{Cl}=1.52-5.33)$.

\section{Discussion}

The frequency of asymptomatic hyperuricemia was 4 times higher in patients with plaque psoriasis than the control group (69 versus $17 \%$ ), higher than that reported by Gisondi in Italy in 2014, who described that it was only 3 -fold higher than in the control group (19 versus $7 \%$ ). ${ }^{8}$ 
Gaceta Médica de México. 2018;154

Table 2. Statistical characteristics and studied variables in patients with plaque psoriasis

\begin{tabular}{|c|c|c|c|c|c|c|}
\hline \multirow{2}{*}{$\frac{\text { Variable }}{\text { Gender (male/female) }}$} & \multicolumn{2}{|c|}{ Normal uric acid $(n=14)$} & \multicolumn{2}{|c|}{ Hyperuricemia $(n=30)$} & \multicolumn{2}{|l|}{$\mathrm{p}$} \\
\hline & \multicolumn{2}{|c|}{$7 / 7$} & \multicolumn{2}{|c|}{$22 / 9$} & 0.08 & $\stackrel{\infty}{-}$ \\
\hline & $\mathrm{n}$ & $\%$ & $\mathrm{n}$ & $\%$ & & $\underline{\underline{n}}$ \\
\hline Arterial hypertension, $\mathrm{n}(\%)$ & 2 & 14.2 & 8 & 35.4 & 0.07 & ¿े \\
\hline Nail alterations, $n(\%)$ & 8 & 57.1 & 18 & 58 & 0.43 & हो \\
\hline Psoriatic arthritis, n (\%) & 4 & 28.5 & 17 & 54.8 & 0.01 & (a) \\
\hline
\end{tabular}

\begin{tabular}{|c|c|c|c|c|}
\hline & Median \pm SD & Median \pm SD & & $\ddot{\omega}$ \\
\hline Age, years & $52.69 \pm 17.62$ & $49.58 \pm 17.54$ & 0.93 & $\overline{\bar{n}}$ \\
\hline Body mass index $\left(\mathrm{kg} / \mathrm{m}^{2}\right)$ & $32.42 \pm 7.32$ & $32.54 \pm 7.66$ & 0.90 & $\frac{3}{0}$ \\
\hline PASI & $5.82 \pm 2.80$ & $10.90 \pm 5.81$ & 0.007 & $\stackrel{\digamma}{\rightleftarrows}$ \\
\hline $\mathrm{BSA}(\%)$ & $15.26 \pm 13.26$ & $28.26 \pm 19.06$ & 0.16 & $\frac{\circ}{\circ}$ \\
\hline PGA & $2.38 \pm 0.76$ & $2.96 \pm 0.76$ & 0.95 & $\frac{\bar{n}}{\rightleftharpoons}$ \\
\hline Total cholesterol (mg/dL) & $196.23 \pm 65.98$ & $193.08 \pm 40.39$ & 0.02 & $\frac{5}{2}$ \\
\hline Triglycerides (mg/dL) & $165.38 \pm 105.28$ & $203.28 \pm 96.6$ & 0.66 & $\frac{\varsigma}{d}$ \\
\hline
\end{tabular}

The differences were analyzed with Student's t-test and the Chi-square test (categorical variables). PASI = Psoriasis Area and Severity Index, BSA = Body Surface Area, PGA = Physician Global Assessment.

The fact that our control population showed a hyperuricemia frequency as high as that of patients with psoriasis in Gisondi's study stands out. ${ }^{8}$ However, our control group had a hyperuricemia frequency similar to that expected in the Mexican population without psoriasis (Llamazares, 19 $\%)^{15}$ This is in agreement with findings described by Li in a 2016 meta-analysis, where the association with hyperuricemia was related to an ethnic component. ${ }^{7}$

In the group of patients with psoriasis there was no statistically significant difference regarding gender, age, body mass index, dyslipidemia and nail alterations between the subgroup with hyperuricemia in comparison with the group with normal uric acid levels of. This is the first study on uric acid conducted in a Mexican population with plaque psoriasis.

As expected, the patients who showed hyperuricemia levels according to their gender had higher PASI-measured severity. This relationship is even higher when the Pearson's logistic correlation coefficient is used. On the other hand, the poor correlation between uric acid and BSA as a clinical severity scale, when compared with the proportional correlation found with PASI, suggests that hyperuricemia pathophysiology not only depends on extensive involvement, but also on specific inflammation characteristics of the disease and, moreover, that uric acid can serve as an objective scale for severity and treatment response assessment.

As regards psoriatic arthritis, reports indicate that uric acid is a serum marker of joint damage in patients with plaque psoriasis and underscore the need for uric acid routine measurement. Therefore, the results of this study indicate that hyperuricemia increases the risk for psoriatic arthritis almost 3-fold.

Even when no relationship was found between serum uric acid and cardiovascular risk in psoriasis, it has been demonstrated in other populations, and further studies with larger samples are therefore suggested. Furthermore, it is necessary to delve into the specific mechanisms of hyperuricemia development in psoriasis and in its usefulness as a predictor cardiovascular and kidney disease in patients with psoriasis, regardless of how modest its clinical expression is.

\section{References}

1. Gudjonsson JE, Elder JT. Psoriasis: Epidemiology. Clin Dermatol. 2007;25(6):535-546.

2. Takeshita J, Grewal S, Langan SM, Mehta NN, Ogdie A, Van-Voorhees AS, et al. Psoriasis and comorbid diseases: Epidemiology. J Am Acad Dermatol. 2017;76(3):377-390.

3. Krueger J, Bowcock A. Psoriasis pathophysiology: Current concepts of pathogenesis. Ann Rheum Dis. 2005:64(Suppl 2):ii30-ii36.

4. Rivas-Bejarano JJ, Valdecantos WC. Psoriasis as autoinflammatory disease. Dermatol Clin. 2013;31(3):445-460.

5. Takeshita J, Grewal S, Langan SM, Mehta NN, Ogdie A, Van-Voorhees AS, et al. Psoriasis and comorbid diseases: implications for management. J Am Acad Dermatol. 2017;76(3):393-403.

6. Kwon HH, Kwon IH, Choi JW, Youn Jl. Cross-sectional study on the correlation of serum uric acid with disease severity in Korean patients with psoriasis. Clin Exp Dermatol. 2011;36:473-478.

7. Lobitz W, Brunsting L. Unpublished studies. En: Ormsby OS, Montgomery $\mathrm{H}$. Diseases of the skin. USA: Lea and Febiger; 1948.

8. Gisondi $P$, Targher G, Cagalli A, Girolomi G. Hyperuricemia in patients with chronic plaque psoriasis. J Am Acad Dermatol. 2014;70(1):127-130.

9. Li X, Miao X, Wang H, Wang Y, Li F, Yang Q, et al. Association of serum uric acid levels in psoriasis: a systematic review and meta-analysis. Medicine (Baltimore). 2016;95(19):e3676. 
10. González-Gay MA, González-Juanatey C, Vázquez-Rodríguez TR, Gómez-Acebo I, Miranda-Filloy JA, Paz-Carreira J, et al. Asymptomatic hyperuricemia and serum uric acid concentration correlate with subclinical atherosclerosis in psoriatic arthritis patients without clinically evident cardiovascular disease. Semin Arthritis Rheum. 2009;39(3):157-162.

11. Isha, Jain VK, Lal H. C-reactive protein and uric acid levels in patients with psoriasis. Indian J Clin Biochem. 2011;26(3):309-311.

12. Henseler T, Schmitt-Rau K. A comparison between BSA, PASI, PLAS and SAPASI as measures of disease severity and improvement by therapy in patients with psoriasis. Int J Dermatol. 2008;47(10):1019-1023.
13. Langley RG, Ellis CN. Evaluating psoriasis with Psoriasis Area and Severity Index, Psoriasis Global Assessment, and Lattice System Physician's Global Assessment. J Am Acad Dermatol. 2004;51(4):563-569.

14. Torre-Alonso JC. Utilidad y aplicación en la práctica clínica de los criterios de CASPAR. Reumatol Clin. 2010;6:18-21.

15. Llamazares-Azuara L, Rodríguez-Martínez M, De-La-Cruz-Mendoza E, Torres-Ruvalcaba A, Flores-Sánchez J. Prevalencia de hiperuricemia, resistencia a la insulina, y dislipidemia en jóvenes de 17 a 23 años. Bioquimia. 2007;32:134. 